\title{
Quasi-Exactly Solvable $N$-Body Spin Hamiltonians with Short-Range Interaction Potentials ${ }^{\star}$
}

\author{
A. ENCISO, F. FINKEL, A. GONZÁLEZ-LÓPEZ * and M.A. RODRÍGUEZ \\ Depto. Física Teórica II, Universidad Complutense, 28040 Madrid, Spain \\ * E-mail: artemio@fis.ucm.es
}

Received September 15, 2006, in final form October 23, 2006; Published online November 03, 2006

Original article is available at http://www.emis.de/journals/SIGMA/2006/Paper073/

\begin{abstract}
We review some recent results on quasi-exactly solvable spin models presenting near-neighbors interactions. These systems can be understood as cyclic generalizations of the usual Calogero-Sutherland models. A nontrivial modification of the exchange operator formalism is used to obtain several infinite families of eigenfunctions of these models in closed form.
\end{abstract}

Key words: Calogero-Sutherland models; exchange operators; quasi-exact solvability

2000 Mathematics Subject Classification: 81Q05; 35Q40

\section{Introduction}

In the early 1970s, F. Calogero [6] and B. Sutherland [37] introduced the quantum integrable systems that nowadays bear their names. Apart from their intrinsic mathematical interest [25, 21, 3], Calogero-Sutherland (CS) models have played a central role in Physics due to their relevant applications to as diverse topics as soliton theory [23, 32], quantum field and string theory [18, 9], quantum Hall effect [2], fractional statistics [28] and random matrix theory [36]. The first satisfactory explanation of the integrability of these models was given by Olshanetsky and Perelomov [26], who connected these models with the radial Laplacian of symmetric spaces associated to the root system $A_{N}$. This unified view enabled them to introduce important generalizations, including different root systems and elliptic potentials.

During the last decade CS models have been extended to the case of particles with internal degrees of freedom, which we shall henceforth call spin. There are two different approaches to spin CS models, namely the supersymmetric [5] and Dunkl operator [10, 29] formalisms. These methods have allowed to solve totally or partially several rational, trigonometric and elliptic spin models, both in their $A_{N}$ and $B C_{N}$ versions $[8,42,16,17]$. The interest in spin CS models also increased as a consequence of their direct connection with the Haldane-Shastry (HS) spin chain [19, 34], which was laid bare by Polychronakos through the so called "freezing trick" [30]. This technique was also used in the construction of solvable spin chains associated to different potentials and root systems [31, 12].

Auberson, Jain and Khare [22, 1] introduced partially solvable versions of the CS models in which each particle only interacts with its nearest and next-to-nearest neighbors. Similar scalar models were also studied by Ezung, Gurappa, Khare and Panigrahi [15]. There are two reasons that make these kind of systems very promising from a physical point of view. First, some of them are related to the short-range Dyson model in random matrix theory [4]. Second,

${ }^{\star}$ This paper is a contribution to the Proceedings of the Workshop on Geometric Aspects of Integrable Systems (July 17-19, 2006, University of Coimbra, Portugal). The full collection is available at http://www.emis.de/journals/SIGMA/Coimbra2006.html 
the HS chains associated to these models would occupy an interesting intermediate position between the Heisenberg chain (short-range, position-independent interactions) and the usual HS chains (long-range, position-dependent interactions). A first step towards the construction of these chains was Deguchi and Ghosh's definition of spin 1/2 versions of the Jain and Khare Hamiltonians [7] using the supersymmetric formalism. Unfortunately, all these authors solely managed to construct a few exact solutions, and all of them with trivial spin dependence, and the procedures developed to obtain exact solutions are by no means systematic.

In $[13,14]$ we introduced three new families of spin near-neighbors models and used a nontrivial modification of the Dunkl operator method to obtain a wide range of fully explicit solutions. In this article we aim to review the main ideas underlying our constructions and explain their connection with the usual CS models. In Section 2 we define the Hamiltonians that we shall deal with and state our main result, which is a description of the algebraic states of the models. In Section 3 we review the philosophy underlying the calculation of exact solutions of Schrödinger's equation by algebraic methods. In Section 4 we sketch the main logical steps that the construction of the invariant flags rests upon. We finish the paper by showing in Section 5 how the actual computation of the algebraic eigenfunctions can be carried out. For the sake of brevity, complete proofs are not given in this review.

\section{Main result}

Let $\Sigma$ be the Hilbert space of internal degrees of freedom of $N$ particles of spin $M \in \frac{1}{2} \mathbb{N}$. Let us fix a basis

$$
\mathcal{B}=\left\{\left|s_{1}, \ldots, s_{N}\right\rangle: s_{i} \in\{-M,-M+1, \ldots, M\}\right\}
$$

of $\Sigma$ and define the spin exchange operators $S_{i j}$ as

$$
S_{i j}\left|s_{1}, \ldots, s_{i}, \ldots, s_{j}, \ldots, s_{N}\right\rangle=\left|s_{1}, \ldots, s_{j}, \ldots, s_{i}, \ldots, s_{N}\right\rangle \text {. }
$$

These operators can be expressed in terms of the (normalized) fundamental generators of $\mathrm{SU}(2 M+1)$ as $S_{i j}=(2 M+1)^{-1}+\sum_{a} J_{i}^{a} J_{j}^{a}$, the index sum ranging from 1 to $4 M(M+1)$.

The Hamiltonians of the models we shall be concerned with are given by (cf. [14])

$$
H_{\epsilon}=-\sum_{i} \partial_{x_{i}}^{2}+V_{\epsilon}, \quad \epsilon=0,1,2
$$

where

$$
\begin{aligned}
V_{0}= & \omega^{2} r^{2}+\sum_{i} \frac{2 a^{2}}{\left(x_{i}-x_{i-1}\right)\left(x_{i}-x_{i+1}\right)}+\sum_{i} \frac{2 a}{\left(x_{i}-x_{i+1}\right)^{2}}\left(a-S_{i, i+1}\right), \\
V_{1}= & \omega^{2} r^{2}+\sum_{i} \frac{b(b-1)}{x_{i}^{2}}+\sum_{i} \frac{8 a^{2} x_{i}^{2}}{\left(x_{i}^{2}-x_{i-1}^{2}\right)\left(x_{i}^{2}-x_{i+1}^{2}\right)} \\
& +4 a \sum_{i} \frac{x_{i}^{2}+x_{i+1}^{2}}{\left(x_{i}^{2}-x_{i+1}^{2}\right)^{2}}\left(a-S_{i, i+1}\right), \\
V_{2}= & 2 a^{2} \sum_{i} \cot \left(x_{i}-x_{i-1}\right) \cot \left(x_{i}-x_{i+1}\right)+2 a \sum_{i} \csc ^{2}\left(x_{i}-x_{i+1}\right)\left(a-S_{i, i+1}\right),
\end{aligned}
$$

with $r^{2}=\sum_{i} x_{i}^{2}$ and $a, b>1 / 2$. Here and in what follows, all sums and products run from 1 to $N$ unless otherwise stated, with the identifications $x_{0} \equiv x_{N}$ and $x_{N+1} \equiv x_{1}$. There is a hyperbolic potential analogous to (2c) that is recovered substituting $x_{i}$ by $-x_{i}$ and $V_{2}$ by $-V_{2}$. The scalar 
reductions $H_{\epsilon}^{\mathrm{sc}}$ of these models are obtained from the above Hamiltonians by the substitution $S_{i, i+1} \rightarrow 1$.

A few remarks on the configuration spaces of these models are now in order. In all three models the potential diverges as $\left(x_{i}-x_{i+1}\right)^{-2}$ on the hyperplanes $x_{i}=x_{i+1}$, so that the particles $i$ and $i+1$ cannot overtake one another. Since we are interested in models with nearest and nextto-nearest neighbors interactions, we shall henceforth assume that $x_{1}<\cdots<x_{N}$. For the second potential $(2 \mathrm{~b})$ we shall take in addition $x_{1}>0$, due to the double pole at $x_{i}=0$.

A first observation concerning the eigenstates of the models (1) is that if $\psi$ is an eigenfunction of $H_{\epsilon}^{\text {sc }}$ with energy $E$, then the factorized state $\Psi=\psi|s\rangle$ is an eigenfunction of $H_{\epsilon}$ with the same energy for any spin state $|s\rangle$ symmetric under permutation of particles. A second observation is that $H_{2}$ commutes with the total momentum $P=-\mathrm{i} \sum_{i} \partial_{x_{i}}$. Hence the movement of the center of mass decouples, and only the eigenfunctions of $\mathrm{H}_{2}$ with zero total momentum need to be considered.

The next theorem summarizes the main results presented in [13, 14], which yield a fully explicit description of several families of algebraic eigenfunctions. It is not difficult to realize, however, that these eigenfunctions do not exhaust the whole spectrum of the models. We shall denote by $\Lambda$ the projection operator on states totally symmetric under the simultaneous permutation of both the spatial and spin coordinates. We define the spin vectors $\left|s_{i}\right\rangle,\left|s_{i j}\right\rangle$ as

$$
\Lambda\left(x_{1}|s\rangle\right)=\sum_{i} x_{i}\left|s_{i}\right\rangle, \quad \Lambda\left(x_{1} x_{2}|s\rangle\right)=\sum_{i<j} x_{i} x_{j}\left|s_{i j}\right\rangle
$$

We shall consider the subspace $\Sigma^{\prime} \subset \Sigma$ of spin vectors $|s\rangle$ such that $\sum_{i}\left|s_{i}\right\rangle$ is symmetric. A thorough characterization of this space is given in [14].

Theorem 1. Let $l, m$ be nonnegative integers and let $|s\rangle$ be an arbitrary spin vector, and denote by $\bar{x}$ the center of mass coordinate $\frac{1}{N} \sum_{i} x_{i}$. Then the following statements hold:

1. Let $\alpha=N\left(a+\frac{1}{2}\right)-\frac{3}{2}, \beta \equiv \beta(m)=1-m-N\left(a+\frac{1}{2}\right), t=\frac{2 r^{2}}{N \bar{x}^{2}}-1$, and $\mu_{l m}=$ $\mathrm{e}^{-\frac{\omega}{2} r^{2}} \bar{x}^{m} L_{l}^{-\beta}\left(\omega r^{2}\right) \prod_{i}\left|x_{i}-x_{i+1}\right|^{a}$. The Hamiltonian $H_{0}$ possesses the following families of spin eigenfunctions with eigenvalue $E_{l m}=E_{0}+2 \omega(2 l+m)$, where $E_{0}=N \omega(2 a+1)$ is the ground state energy:

$$
\begin{aligned}
\Psi_{l m}^{(0)}= & \mu_{l m} P_{\left[\frac{m}{2}\right]}^{(\alpha, \beta)}(t) \Lambda|s\rangle, \quad m \geq 0, \\
\Psi_{l m}^{(1)}= & \mu_{l, m-1}\left[P_{\left[\frac{m-1}{2}\right]}^{(\alpha+1, \beta)}(t) \Lambda\left(x_{1}|s\rangle\right)-\bar{x} P_{\left[\frac{m-1}{2}\right]}^{(\alpha+1, \beta)}(t) \Lambda|s\rangle\right], \quad m \geq 1, \\
\Psi_{l m}^{(2)}= & \mu_{l, m-2}\left[P_{\left[\frac{m}{2}\right]-1}^{(\alpha+2, \beta)}(t)\left(\Lambda\left(x_{1}^{2}|s\rangle\right)-2 \bar{x} \Lambda\left(x_{1}|s\rangle\right)\right)\right. \\
& \left.+\bar{x}^{2}\left(P_{\left[\frac{m}{2}\right]-1}^{(\alpha+2, \beta)}(t)-\frac{2(\alpha+1)}{2\left[\frac{m-1}{2}\right]+1} P_{\left[\frac{m}{2}\right]-1}^{(\alpha+1, \beta)}(t)\right) \Lambda|s\rangle\right], \quad m \geq 2, \\
\Psi_{l m}^{(3)}= & \mu_{l, m-3}\left[\frac{2}{3 N} P_{\left[\frac{m-3}{2}\right]}^{(\alpha+3, \beta)}(t) \sum_{i} x_{i}^{3}+\bar{x}^{3} \varphi_{m}(t)\right] \Lambda|s\rangle, \quad m \geq 3, \\
\Psi_{l m}^{(4)}= & \mu_{l, m-4}\left[\frac{3}{2\left(\left[\frac{m-3}{2}\right]+\frac{1}{2}\right)} \bar{x}^{2} P_{\left[\frac{m}{2}\right]-2}^{(\alpha+3, \beta)}(t) \Lambda\left(x_{1}^{2}|s\rangle\right)\right. \\
& +\left(\frac{3}{2} \bar{x}^{3} \phi_{m}(t)-\frac{1}{N} P_{\left[\frac{m}{2}\right]-2}^{(\alpha+4, \beta)}(t) \sum_{i} x_{i}^{3}\right) \Lambda\left(x_{1}|s\rangle\right) \\
& \left.+\left(\frac{1}{N} \bar{x} P_{\left[\frac{m}{2}\right]-2}^{(\alpha+4, \beta)}(t) \sum_{i} x_{i}^{3}+\frac{3}{2} \bar{x}^{4} \chi_{m}(t)\right) \Lambda|s\rangle\right], \quad m \geq 4 .
\end{aligned}
$$


When $|s\rangle \in \Sigma^{\prime}$ there is an additional family of eigenfunctions given by

$$
\begin{aligned}
\widetilde{\Psi}_{l m}^{(2)}= & \mu_{l, m-2}\left[P_{\left[\frac{m}{2}\right]-1}^{(\alpha+2, \beta)}(t)\left(\Lambda\left(x_{1} x_{2}|s\rangle\right)-2 \bar{x} \Lambda\left(x_{1}|s\rangle\right)\right)\right. \\
& \left.+\bar{x}^{2}\left(P_{\left[\frac{m}{2}\right]-1}^{(\alpha+2, \beta)}(t)+\frac{2(\alpha+1)}{\left(2\left[\frac{m-1}{2}\right]+1\right)(N-1)} P_{\left[\frac{m}{2}\right]-1}^{(\alpha+1, \beta)}(t)\right) \Lambda|s\rangle\right], \quad m \geq 2 .
\end{aligned}
$$

The functions $\varphi_{m}, \phi_{m}$ and $\chi_{m}$ are polynomials given explicitly by

$$
\begin{aligned}
\varphi_{m}= & \frac{m+2 \alpha+2}{m-1} P_{\frac{m}{2}}^{(\alpha+2, \beta-2)}-P_{\frac{m}{2}-1}^{(\alpha+3, \beta-1)}-\frac{4 \alpha+7}{m-1} P_{\frac{m}{2}-1}^{(\alpha+2, \beta-1)}+\frac{1}{3} P_{\frac{m}{2}-2}^{(\alpha+3, \beta)}, \\
\phi_{m}= & P_{\frac{m}{2}-1}^{(\alpha+4, \beta-1)}-2 P_{\frac{m}{2}-1}^{(\alpha+3, \beta-1)}-\frac{m+2 \alpha+3}{(m-1)(m-3)} P_{\frac{m}{2}-1}^{(\alpha+2, \beta-1)} \\
& -\frac{1}{3} P_{\frac{m}{2}-2}^{(\alpha+4, \beta)}+\frac{m+2 \alpha-1}{m-3} P_{\frac{m}{2}-2}^{(\alpha+3, \beta)}, \\
\chi_{m}= & \frac{3 m+2 \alpha}{(m-1)(m-3)} P_{\frac{m}{2}-1}^{(\alpha+2, \beta-1)}+\frac{2 m-7}{m-3} P_{\frac{m}{2}-1}^{(\alpha+3, \beta-1)}-P_{\frac{m}{2}-1}^{(\alpha+4, \beta-1)} \\
& -\frac{m+2 \alpha+2}{(m-1)(m-3)} P_{\frac{m}{2}-2}^{(\alpha+2, \beta)}-\frac{m+2 \alpha}{m-3} P_{\frac{m}{2}-2}^{(\alpha+3, \beta)}+\frac{1}{3} P_{\frac{m}{2}-2}^{(\alpha+4, \beta)},
\end{aligned}
$$

for even $m$, and

$$
\begin{aligned}
\varphi_{m}= & 2 P_{\frac{m-1}{2}}^{(\alpha+2, \beta-1)}-P_{\frac{m-1}{2}}^{(\alpha+3, \beta-1)}+\frac{1}{3} P_{\frac{m-3}{2}}^{(\alpha+3, \beta)}+\frac{m+2 \alpha+2}{m(m-2)} P_{\frac{m-3}{2}}^{(\alpha+1, \beta)} \\
& -\frac{m+2 \alpha+2}{m-2} P_{\frac{m-3}{2}}^{(\alpha+2, \beta)}, \\
\phi_{m}= & P_{\frac{m-3}{2}}^{(\alpha+4, \beta-1)}-\frac{2 m-5}{m-2} P_{\frac{m-3}{2}}^{(\alpha+3, \beta)}-\frac{1}{3} P_{\frac{m-5}{2}}^{(\alpha+4, \beta)}+\frac{m+2 \alpha-1}{m-2} P_{\frac{m-5}{2}}^{(\alpha+3, \beta)}, \\
\chi_{m}= & \frac{2 m-3}{m(m-2)} P_{\frac{m-3}{2}}^{(\alpha+2, \beta-1)}+\frac{2(m-3)}{m-2} P_{\frac{m-3}{2}}^{(\alpha+3, \beta-1)}-P_{\frac{m-3}{2}}^{(\alpha+4, \beta-1)} \\
& -\frac{m+2 \alpha+1}{m(m-2)} P_{\frac{m-5}{2}}^{(\alpha+2, \beta)}-\frac{m+2 \alpha}{m-2} P_{\frac{m-5}{2}}^{(\alpha+3, \beta)}+\frac{1}{3} P_{\frac{m-3}{2}}^{(\alpha+4, \beta)},
\end{aligned}
$$

for odd $m$.

2. The Hamiltonian $H_{1}$ possesses the following families of spin eigenfunctions with eigenvalue $E_{k}=E_{0}+4 k \omega$, where $E_{0}=N \omega(4 a+2 b+1)$ is the ground state energy:

$$
\begin{aligned}
\Psi_{k}^{(0)} & =\mu L_{k}^{\alpha-1}\left(\omega r^{2}\right) \Lambda|s\rangle, \quad k \geq 0, \\
\Psi_{k}^{(1)} & =\mu L_{k-1}^{\alpha+1}\left(\omega r^{2}\right)\left[N \Lambda\left(x_{1}^{2}|s\rangle\right)-r^{2} \Lambda|s\rangle\right], \quad k \geq 1, \\
\Psi_{k}^{(2)} & =\mu L_{k-2}^{\alpha+3}\left(\omega r^{2}\right)\left[N(\alpha+1) \sum_{i} x_{i}^{4}-\beta r^{4}\right] \Lambda|s\rangle, \quad k \geq 2,
\end{aligned}
$$

with $\alpha=N\left(2 a+b+\frac{1}{2}\right), \beta=N\left(4 a+b+\frac{3}{2}\right)$ and $\mu=\mathrm{e}^{-\frac{\omega}{2} r^{2}} \prod_{i}\left|x_{i}^{2}-x_{i+1}^{2}\right|^{a} x_{i}^{b}$.

3. The Hamiltonian $\mathrm{H}_{2}$ possesses the following spin eigenfunctions with zero momentum

$$
\begin{aligned}
& \Psi_{0}=\mu \Phi^{(0)}, \quad \Psi_{1,2}=\mu \sum_{i}\left\{\begin{array}{l}
\cos \\
\sin
\end{array}\right\}\left(2\left(x_{i}-\bar{x}\right)\right)\left|s_{i}\right\rangle, \\
& \Psi_{3}=\mu\left[\frac{2 a}{2 a+1} \Phi^{(0)}+\sum_{i \neq j} \cos \left(2\left(x_{i}-x_{j}\right)\right)\left|s_{j}\right\rangle\right], \quad \Psi_{4}=\mu \sum_{i \neq j} \sin \left(2\left(x_{i}-x_{j}\right)\right)\left|s_{j}\right\rangle,
\end{aligned}
$$

where $\mu=\prod_{i} \sin ^{a}\left|x_{i}-x_{i+1}\right|$. Their energies are respectively given by

$$
E_{0}, \quad E_{1,2}=E_{0}+4\left(2 a+1-\frac{1}{N}\right), \quad E_{3,4}=E_{0}+8(2 a+1),
$$

where $E_{0}=2 N a^{2}$ is the ground state energy. 


\section{Calogero models and Dunkl operators}

Let us consider a self-adjoint operator $H$ acting on a given Hilbert space $\mathcal{H}$. The idea underlying the construction of algebraic eigenfunctions of $H$ (see, e.g., [40]) is that the explicit knowledge of a finite-dimensional subspace $\mathcal{H}_{1} \subset \mathcal{H}$ which is invariant under the operator $H$ allow ones to compute $\operatorname{dim} \mathcal{H}_{1}$ eigenfunctions and eigenvalues of $H$ by algebraic methods, i.e., diagonalizing the matrix $\left.H\right|_{\mathcal{H}_{1}}$. In this case the operator is said to be quasi-exactly solvable (QES). In fact, the Hamiltonians of the models (2a) and (2b) possess an infinite flag $\mathcal{H}_{1} \subset \mathcal{H}_{2} \subset \cdots$ of known finite-dimensional invariant subspaces, which yield an arbitrary large number of eigenvalues and eigenfunctions. Although such models are sometimes termed "exactly solvable" [39], we will not use this terminology since the algebraic eigenfunctions of the models $(2 \mathrm{a})$ and $(2 \mathrm{~b})$ are not an orthonormal basis of the Hilbert space.

A particularly convenient method of carrying out this program [38, 35] is through a Lie algebra $\mathfrak{g}$ of first-order differential operators $J^{a}=\sum_{i} \xi^{a i}(\boldsymbol{z}) \partial_{z_{i}}+\eta^{a}(\boldsymbol{z})(a=1, \ldots, r)$ acting on a finite-dimensional module $\mathcal{M} \subset C^{\infty}(M)$, with $M$ a domain in $\mathbb{R}^{N}$. Let us assume that there exists a second-order differential operator $\tilde{H}=\sum_{a, b=1}^{r} c_{a b} J^{a} J^{b}+\sum_{a=1}^{r} c_{a} J^{a}+c_{0}$ that is equivalent, up to gauge transformation $\tilde{H} \mapsto \mu \tilde{H} \mu^{-1}\left(\mu \in C^{\infty}\left(M, \mathbb{R}^{+}\right)\right)$and global change of variables $\boldsymbol{z} \in$ $M \mapsto \boldsymbol{x} \in \mathbb{R}^{N}$, to a Schrödinger operator $H=-\Delta_{g}+V, \Delta_{g}$ standing for the Laplace-Beltrami operator in $\left(\mathbb{R}^{N}, g\right)$. If $\left.\mu \mathcal{M}\right|_{\boldsymbol{z} \mapsto \boldsymbol{x}} \subset L^{2}\left(\mathbb{R}^{N}, \sqrt{g} \mathrm{~d} \boldsymbol{x}\right)$, then one can obtain $\operatorname{dim} \mathcal{M}$ eigenfunctions of $H$ by algebraic methods. The Schrödinger operators amenable to this treatment are termed Lie-algebraic. It should be observed that the operators $J^{a}$ are not symmetries of $H$ : $\mathfrak{g}$ is an algebra of hidden symmetries of $H$.

Dunkl operators [20] were originally introduced to study spherical harmonics associated to measures invariant under a Coxeter group [10]. Actually, let $\boldsymbol{v}, \boldsymbol{z} \in \mathbb{R}^{N}$ and define the reflection $\sigma_{\boldsymbol{v}} \boldsymbol{z}=\boldsymbol{z}-2|\boldsymbol{v}|^{-2}(\boldsymbol{z} \cdot \boldsymbol{v}) \boldsymbol{v}$. Let $\mathfrak{W}$ be a Coxeter group, which can be assumed to be the Weyl group of a (possibly nonreduced) root system $R$, and let $\boldsymbol{v}_{a}=\sum_{i} v_{a}^{i} \boldsymbol{e}_{i}(a=1, \ldots, r)$ be a basis of positive roots. Define an action of $\mathfrak{W}$ on $\mathbb{C}[\boldsymbol{z}]$ as $K_{a} f=f \circ \sigma_{\boldsymbol{v}_{a}}$. Dunkl operators were originally defined as

$$
J_{i}=\partial_{z_{i}}+\sum_{a=1}^{r} \frac{g_{a}^{2} v_{a}^{i}}{\boldsymbol{z} \cdot \boldsymbol{v}_{a}}\left(1-K_{a}\right),
$$

where the real parameters $g_{a}^{2}$ are chosen so that they are constant on each orbit of $\mathfrak{W}$. The operators $J_{i}$ can be understood as deformations of the partial derivatives $\partial_{z_{i}}$ that commute with the deformed Laplacian, i.e., $\sum_{i} J_{i}^{2} J_{j}=J_{j} \sum_{i} J_{i}^{2}$. It can be verified that $\left\{J_{i}, K_{a}\right\}$ span a degenerate Hecke algebra [24]. Currently, the definition of Dunkl operators has been generalized to mean a set $\left\{J_{i}\right\}_{i=1}^{N} \subset$ End $\mathbb{C}[\boldsymbol{z}]$ of first order differential operators which leave invariant finitedimensional polynomial subspaces and such that $\left\{J_{i}, K_{a}\right\}$ span a Hecke algebra.

In their original form, Dunkl operators are directly connected with the rational Calogero model of type $R$ [11]. In the simplest case $\left(R=A_{N-1}, \mathfrak{W}=S_{N}\right)$ and writing $\boldsymbol{x}$ instead of $\boldsymbol{z}$, the Dunkl operators (3) read

$$
J_{i}=\partial_{x_{i}}+g^{2} \sum_{j \neq i} \frac{1}{x_{i}-x_{j}}\left(1-K_{i j}\right),
$$

where

$$
\left(K_{i j} f\right)\left(x_{1}, \ldots, x_{i}, \ldots, x_{j}, \ldots, x_{N}\right)=f\left(x_{1}, \ldots, x_{j}, \ldots, x_{i}, \ldots, x_{N}\right)
$$

denotes the reflection operator associated to the root $\boldsymbol{e}_{i}-\boldsymbol{e}_{j}$. Actually, let us consider the 
Calogero ground state function $\mu(\boldsymbol{x})=\mathrm{e}^{-\omega r^{2}} \prod_{i<j}\left|x_{i}-x_{j}\right|^{a}$ and the auxiliary operator

$$
J^{0}=\sum_{i} x_{i} \partial_{x_{i}}
$$

A straightforward calculation shows that one can use the ground state function $\mu$ to gauge transform the deformed Laplacian so that

$$
\mu \bar{H}_{\mathrm{C}} \mu^{-1}=-\sum_{i} \partial_{x_{i}}^{2}+a \sum_{i \neq j} \frac{1}{\left(x_{i}-x_{j}\right)^{2}}\left(a-K_{i j}\right)+\omega r^{2},
$$

with $g^{2}=a(a-1)$ and

$$
\bar{H}_{\mathrm{C}}=\sum_{i} J_{i}^{2}+2 \omega J^{0}+E_{0}
$$

yields the Calogero model of $A_{N-1}$ type when acting on symmetric functions $\psi \in \Lambda L^{2}\left(\mathbb{R}^{N}\right)$.

Since $J_{i}, J^{0}$ preserve the space of polynomials

$$
\mathcal{P}^{n}=\{f \in \mathbb{C}[\boldsymbol{x}]: \operatorname{deg} f \leq n\}
$$

for any $n=0,1, \ldots$, the Dunkl operators provide a very convenient fashion of exploring the solvability properties of Calogero-Sutherland models. It should be remarked, however, that for an arbitrary Coxeter group $\mathfrak{W}$ the Dunkl operators (3) do not form a Lie algebra; nevertheless, this technique captures most of the relevant features of the Lie-algebraic method.

As we shall now outline, Dunkl operators can also be used to introduce internal degrees of freedom in the picture without breaking the solvability properties of the models. Given a scalar differential-difference operator $D$ linear in $K_{i j}$, let us denote by $D^{*}$ the differential operator acting on $C^{\infty} \otimes \Sigma$ obtained from $D$ by the replacement $K_{i j} \rightarrow S_{i j}$. It is clear that the actions of $D$ and $D^{*}$ coincide on the (bosonic) Hilbert space

$$
\mathcal{H}=\Lambda\left(L^{2}\left(\mathbb{R}^{N}\right) \otimes \Sigma\right) .
$$

Therefore the scalar operator (6) coincides with that of the spin Calogero model

$$
H_{\mathrm{C}} \equiv \mu \bar{H}_{\mathrm{C}}^{*} \mu^{-1}=-\sum_{i} \partial_{x_{i}}^{2}+a \sum_{i \neq j} \frac{1}{\left(x_{i}-x_{j}\right)^{2}}\left(a-S_{i j}\right)
$$

when acting on symmetric states $\Psi \in \mathcal{H}$.

\section{Invariant subspaces}

The proof of the main theorem rests on the construction of appropriate invariant flags for the Hamiltonians (1) using a modification of the Dunkl operator formalism. This modification turns out to be rather nontrivial, ultimately due to the fact the cyclic group is not of Coxeter type.

As the first step, we consider the second-order differential-difference operators $T_{\epsilon}$ given by

$$
T_{\epsilon}=\sum_{i} z_{i}^{\epsilon} \partial_{i}^{2}+2 a \sum_{i} \frac{1}{z_{i}-z_{i+1}}\left(z_{i}^{\epsilon} \partial_{i}-z_{i+1}^{\epsilon} \partial_{i+1}\right)-2 a \sum_{i} \frac{\vartheta_{\epsilon}\left(z_{i}, z_{i+1}\right)}{\left(z_{i}-z_{i+1}\right)^{2}}\left(1-K_{i, i+1}\right),
$$

where $\partial_{i}=\partial_{z_{i}}, z_{N+1} \equiv z_{1}$, and

$$
\vartheta_{0}(x, y)=1, \quad \vartheta_{1}(x, y)=\frac{1}{2}(x+y), \quad \vartheta_{2}(x, y)=x y .
$$


Each Hamiltonian $H_{\epsilon}$ is related to a linear combination

$$
\bar{H}_{\epsilon}=c T_{\epsilon}+c_{-} J^{-}+c_{0} J^{0}+E_{0}
$$

of its corresponding operator $T_{\epsilon}$ and the auxiliary first-order differential operators $J^{-}=\sum_{i} \partial_{i}$ and $J^{0}=\sum_{i} z_{i} \partial_{i}$ via a change of variables, a gauge transformation, and the star mapping defined in the previous section, that is,

$$
H_{\epsilon}=\left.\mu \cdot \bar{H}_{\epsilon}^{*}\right|_{z_{i}=\zeta\left(x_{i}\right)} \cdot \mu^{-1} .
$$

The constants $c, c_{-}, c_{0}, E_{0}$, the gauge factor $\mu$, and the change of variables $\zeta$ for each model are listed in Table 1. Hence the construction of the models (1) is analogous to that of the usual Calogero-Sutherland models, with the operators (9) being a cyclic analog of the sum of the squares of the Dunkl operators.

Table 1. Parameters, gauge factor and change of variable in equations (10) and (11).

\begin{tabular}{lccc}
\hline & $\epsilon=0$ & $\epsilon=1$ & $\epsilon=2$ \\
\hline$c$ & -1 & -4 & 4 \\
\hline$c_{-}$ & 0 & $-2(2 b+1)$ & 0 \\
\hline$c_{0}$ & $2 \omega$ & $4 \omega$ & $4(1-2 a)$ \\
\hline$E_{0}$ & $N \omega(2 a+1)$ & $N \omega(4 a+2 b+1)$ & $2 N a^{2}$ \\
\hline$\mu(\boldsymbol{x})$ & $\mathrm{e}^{-\frac{\omega}{2} r^{2}} \prod_{i}\left|x_{i}-x_{i+1}\right|^{a}$ & $\mathrm{e}^{-\frac{\omega}{2} r^{2}} \prod_{i}\left|x_{i}^{2}-x_{i+1}^{2}\right|^{a} x_{i}^{b}$ & $\prod_{i} \sin ^{a}\left|x_{i}-x_{i+1}\right|$ \\
\hline$\zeta(x)$ & $x$ & $x^{2}$ & $\mathrm{e}^{ \pm 2 \mathrm{i} x}$ \\
\hline
\end{tabular}

It is obvious that the operators (10) preserve the polynomial space (7) for any $n \in \mathbb{N}$, so one may be tempted to believe that the usual arguments for spin CS models should yield an invariant flag for the Hamiltonians (1). Nevertheless, this is not the case. In fact, the standard construction $[16,17]$ is based on the fact that the actions of $H_{\mathrm{C}}$ and $\mu \bar{H}_{\mathrm{C}} \mu^{-1}$ on symmetric states coincide and these operators commute with the $S_{N}$ symmetrizer $\Lambda$. Unfortunately, $H_{\epsilon}$ (or $\bar{H}_{\epsilon}$ ) do not commute with $\Lambda$ and cyclic symmetry does not suffice to exchange $H_{\epsilon}$ and $\mu \bar{H}_{\epsilon} \mu^{-1}$, so this procedure does not grant the existence of any nontrivial invariant subspaces, not even of direct products $\mathcal{M} \otimes \Lambda \Sigma\left(\mathcal{M} \subset C^{\infty}\left(\mathbb{R}^{N}\right)\right)$.

We shall now review the actual construction of the invariant spaces, which is considerably more involved. We shall not provide complete proofs, but merely a sketch of the main logical steps the construction rests upon.

Classical results on the theory of invariants [41] are responsible for the success of studying CS models through symmetric polynomials $[27,33]$. We shall extend this approach to deal with the Hamiltonians (1). Let us first introduce two bases $\left\{\sigma_{k}\right\}$ and $\left\{\tau_{k}\right\}$ of the space of symmetric polynomials in $\boldsymbol{z}$ :

$$
\sigma_{k}=\sum_{i} z_{i}^{k}, \quad \tau_{k}=\sum_{i_{1}<\cdots<i_{k}} z_{i_{1}} \cdots z_{i_{k}} ; \quad k=1, \ldots, N .
$$

The operators $T_{\epsilon}$ consist of three summands which are of second, first and zeroth order in the derivatives. Let us denote each summand by $L_{\epsilon}, 2 a X_{\epsilon}$ and $-2 a A_{\epsilon}$ respectively. It is not difficult to realize that $\left\{L_{\epsilon}\right\}$ span a Lie algebra isomorphic to $\mathfrak{s l}(2)$, as in the CS case. It turns out that the first-order differential operators $X_{\epsilon}$ leave invariant a flag of symmetric polynomials, as stated in the following easy lemma. 
Lemma 1. For each $n=0,1, \ldots$, the operator $X_{\epsilon}$ leaves invariant the linear space $\mathcal{X}_{\epsilon}^{n}$, where $\mathcal{X}_{0}^{n}=\mathbb{C}\left[\sigma_{1}, \sigma_{2}, \sigma_{3}\right] \cap \mathcal{P}^{n}, \quad \mathcal{X}_{1}^{n}=\mathbb{C}\left[\sigma_{1}, \sigma_{2}, \tau_{N}\right] \cap \mathcal{P}^{n}, \quad \mathcal{X}_{2}^{n}=\mathbb{C}\left[\sigma_{1}, \tau_{N-1}, \tau_{N}\right] \cap \mathcal{P}^{n}$.

Remark 1. It should be noted that these flags cannot be trivially enlarged, since, e.g.,

$$
\begin{array}{ll}
\frac{1}{4} X_{0} \sigma_{4}=2 \sigma_{2}+\sum_{i} z_{i} z_{i+1}, & \\
\frac{1}{3} X_{1} \sigma_{3}=2 \sigma_{2}+\sum_{i} z_{i} z_{i+1}, \quad X_{1} \tau_{N-1}=\tau_{N} \sum_{i}\left(z_{i} z_{i+1}\right)^{-1} \\
\frac{1}{2} X_{2} \sigma_{2}=2 \sigma_{2}+\sum_{i} z_{i} z_{i+1}, \quad X_{2} \tau_{N-2}=N \tau_{N-2}-\tau_{N} \sum_{i}\left(z_{i} z_{i+1}\right)^{-1}
\end{array}
$$

are not symmetric polynomials.

In the next proposition we characterize subspaces of the flags described in Lemma 1 that are preserved by the whole operator $T_{\epsilon}$. If $f \in \mathbb{C}\left[\sigma_{1}, \sigma_{2}, \sigma_{3}, \tau_{N-1}, \tau_{N}\right]$, we adopt the convenient notation

$$
f_{k}= \begin{cases}\partial_{\sigma_{k}} f, & k=1,2,3, \\ \partial_{\tau_{k}} f, & k=N-1, N .\end{cases}
$$

Proposition 1. For each $n=0,1, \ldots$, the operator $T_{\epsilon}$ leaves invariant the linear space $\mathcal{S}_{\epsilon}^{n}$, where

$$
\begin{aligned}
& \mathcal{S}_{0}^{n}=\left\{f \in \mathcal{X}_{0}^{n} \mid f_{33}=0\right\}, \\
& \mathcal{S}_{1}^{n}=\left\{f \in \mathcal{X}_{1}^{n} \mid f_{22}=f_{N N}=0\right\}, \\
& \mathcal{S}_{2}^{n}=\left\{f \in \mathcal{X}_{2}^{n} \mid f_{11}=f_{N-1, N-1}=0\right\} .
\end{aligned}
$$

Proposition 1 implies that each operator $T_{\epsilon}$ preserves product symmetric subspaces $\mathcal{S}_{\epsilon}^{n} \otimes \Lambda \Sigma$ spanned by factorized states. The main result on invariant subspaces shows that in fact the latter operator leaves invariant a richer flag of nontrivial finite-dimensional subspaces of $\Lambda\left(\mathcal{P}^{n} \otimes \Sigma\right)$.

\section{Theorem 2. Let}

$$
\begin{aligned}
& \mathcal{T}_{0}^{n}=\left\langle f\left(\sigma_{1}, \sigma_{2}, \sigma_{3}\right) \Lambda \mid s\right\rangle, g\left(\sigma_{1}, \sigma_{2}, \sigma_{3}\right) \Lambda\left(z_{1}|s\rangle\right), h\left(\sigma_{1}, \sigma_{2}\right) \Lambda\left(z_{1}^{2}|s\rangle\right), \tilde{h}\left(\sigma_{1}, \sigma_{2}\right) \Lambda\left(z_{1} z_{2}\left|s^{\prime}\right\rangle\right)\left|f_{33}=g_{33}=0\right\rangle, \\
& \mathcal{T}_{1}^{n}=\left\langle f\left(\sigma_{1}, \sigma_{2}, \tau_{N}\right) \Lambda \mid s\right\rangle, g\left(\sigma_{1}, \tau_{N}\right) \Lambda\left(z_{1}|s\rangle\right)\left|f_{22}=f_{N N}=g_{N N}=0\right\rangle, \\
& \mathcal{T}_{2}^{n}=\left\langle f\left(\sigma_{1}, \tau_{N-1}, \tau_{N}\right) \Lambda \mid s\right\rangle, g\left(\tau_{N-1}, \tau_{N}\right) \Lambda\left(z_{1}|s\rangle\right), \tau_{N} q\left(\sigma_{1}, \tau_{N}\right) \Lambda\left(z_{1}^{-1}|s\rangle\right) \\
& \quad\left|f_{11}=f_{N-1, N-1}=g_{N-1, N-1}=q_{11}=0\right\rangle,
\end{aligned}
$$

where $|s\rangle \in \Sigma,\left|s^{\prime}\right\rangle \in \Sigma^{\prime}, \operatorname{deg} f \leq n, \operatorname{deg} g \leq n-1, \operatorname{deg} h \leq n-2, \operatorname{deg} \tilde{h} \leq n-2, \operatorname{deg} q \leq n-N+1$, and $\operatorname{deg}$ is the total degree in $\bar{z}$. Then $\mathcal{T}_{\epsilon}^{n}$ is invariant under $T_{\epsilon}$ for all $n=0,1, \ldots$

From this theorem one easily obtains the following corollary, which is crucial for the computation of the algebraic eigenfunctions of the models (1).

Corollary 1. For each $\epsilon=0,1,2$, the gauge Hamiltonian $\bar{H}_{\epsilon}$ leaves invariant the space $\overline{\mathcal{H}}_{\epsilon}^{n}$ defined by

$$
\overline{\mathcal{H}}_{0}^{n}=\mathcal{T}_{0}^{n}, \quad \overline{\mathcal{H}}_{1}^{n}=\left.\mathcal{T}_{1}^{n}\right|_{f_{N}=g_{N}=0}, \quad \overline{\mathcal{H}}_{2}^{n}=\mathcal{T}_{2}^{n} .
$$




\section{Spectrum and eigenfunctions}

As happens with the usual CS models, the algebraic eigenvalues of the Hamiltonians (1) can be easily obtained by choosing a basis of the invariant spaces $\mu \overline{\mathcal{H}}_{\epsilon}^{n}$ in which the action of $H_{\epsilon}$ is triangular. In all three cases, the algebraic eigenvalue $E_{0}$ is the ground state energy, since the corresponding eigenfunctions do not vanish in the configuration space $C_{\epsilon}$.

Now we shall outline how the algebraic eigenfunctions in Theorem 1 were calculated. The easiest case is $\epsilon=2$, since states related by a multiplicative factor $\tau_{N}^{k}$ only differ by the movement of the center of mass, which is conserved. Hence the invariant subspaces $\overline{\mathcal{H}}_{2}^{n}$ solely allow one to compute five eigenfunctions of zero total momentum, which are the ones listed in the main theorem.

In the case $\epsilon=1$ the eigenvalue equation reads

$$
\left(\bar{H}_{1}-E_{0}-4 \omega n\right) \Phi=0
$$

where $\Phi$ is a symmetric vector-valued polynomial in $\overline{\mathcal{H}}_{1}^{n}$ of degree $n$. Setting $t=\omega \sigma_{1}$ and $\Phi=\left[p(t)+\sigma_{2} q(t)\right] \Lambda|s\rangle+g(t) \Lambda\left(z_{1}|s\rangle\right)$, this equation can be written as

$$
\mathcal{L}_{k-1}^{\alpha+1} g=\mathcal{L}_{k-2}^{\alpha+3} q=0, \quad \mathcal{L}_{k}^{\alpha-1} p=-\frac{\alpha}{N \omega} g-\frac{2 \beta}{N \omega^{2}} t q,
$$

with the Laguerre operator $\mathcal{L}_{\nu}^{\lambda}$ defined as

$$
\left(\mathcal{L}_{\nu}^{\lambda} f\right)(t)=t f^{\prime \prime}(t)+(\lambda+1-t) f^{\prime}(t)+\nu f(t) .
$$

With some effort one can obtain all the solutions of these equations, as shown in the following proposition.

Proposition 2. The polynomial solutions of the system of ODE's (13) are spanned by

$$
\begin{array}{ll}
\Phi_{n}^{(0)}=L_{n}^{\alpha-1}(t) \Lambda|s\rangle, \quad n \geq 0 \\
\Phi_{n}^{(1)}=L_{n-1}^{\alpha+1}(t)\left[N \omega \Lambda\left(z_{1}|s\rangle\right)-t \Lambda\left(z_{1}|s\rangle\right)\right], & n \geq 1, \\
\Phi_{n}^{(2)}=L_{n-2}^{\alpha+3}(t)\left[N \omega^{2}(\alpha+1) \sigma_{2}-\beta t^{2}\right] \Lambda|s\rangle, & n \geq 2 .
\end{array}
$$

These solutions are characterized by the conditions $q=g=0, q=0$ and $g=0$ respectively and correspond to the algebraic eigenfunctions of $H_{1}$ presented in Theorem 1.

The case $\epsilon=0$ is similar, but the computations become more involved due to the rich structure of the invariant flag. Writing

$$
\Phi=\left(p+\sigma_{3} q\right) \Lambda|s\rangle+\left(u+\sigma_{3} v\right) \Lambda\left(z_{1}|s\rangle\right)+h \Lambda\left(z_{1}^{2}|s\rangle\right)+\tilde{h} \Lambda\left(x_{1} x_{2}|s\rangle\right),
$$

where $\operatorname{deg} \Phi=k$ and $\tilde{h}=0$ if $|s\rangle \notin \Sigma^{\prime}$, the equation $\left(H_{0}-E_{0}-2 \omega k\right) \Phi=0$ reduces to the systems of PDE's

$$
\begin{aligned}
& {\left[L_{0}-2 \omega(k-2)\right] \tilde{h}-8 \tilde{h}_{2}=0} \\
& {\left[L_{0}-2 \omega(k-2)\right] h-8 h_{2}=6 v} \\
& {\left[L_{0}-2 \omega(k-1)\right] u-4 u_{2}=4 h_{1}+4 \tilde{h}_{1}+6 \sigma_{2} v_{1}+6(2 a+1) \sigma_{1} v,} \\
& {\left[L_{0}-2 \omega(k-4)\right] v-16 v_{2}=0} \\
& \left(L_{0}-2 \omega k\right) p=2 u_{1}+2(2 a+1) h-\frac{4 a}{N-1} \tilde{h}+6 \sigma_{2} q_{1}+6(2 a+1) \sigma_{1} q, \\
& {\left[L_{0}-2 \omega(k-3)\right] q-12 q_{2}=2 v_{1}}
\end{aligned}
$$


with

$$
L_{0}=-\left(N \partial_{\sigma_{1}}^{2}+4 \sigma_{1} \partial_{\sigma_{1}} \partial_{\sigma_{2}}+4 \sigma_{2} \partial_{\sigma_{2}}^{2}+2(2 a+1) N \partial_{\sigma_{2}}\right)+2 \omega\left(\sigma_{1} \partial_{\sigma_{1}}+2 \sigma_{2} \partial_{\sigma_{2}}\right) .
$$

One can check by inspection that the system (14) possesses six families of polynomial solutions, as collected in Table 2. These correspond to the six families of algebraic eigenfunctions listed in Theorem 1.

Table 2. The six types of polynomial solutions of the system (14) and their corresponding eigenfunctions.

\begin{tabular}{ll}
\hline \multicolumn{1}{c}{ Conditions } & Corresponding eigenfunction \\
\hline$q=u=v=h=\tilde{h}=0, \quad p \neq 0$ & $\Psi_{l m}^{(0)}$ \\
\hline$u=v=h=\tilde{h}=0, \quad q \neq 0$ & $\Psi_{l m}^{(3)}$ \\
\hline$q=v=h=\tilde{h}=0, \quad u \neq 0$ & $\Psi_{l m}^{(1)}$ \\
\hline$q=v=\tilde{h}=0, \quad h \neq 0$ & $\Psi_{l m}^{(2)}$ \\
\hline$q=v=h=0, \quad \tilde{h} \neq 0$ & $\widetilde{\Psi}_{l m}^{(2)}$ \\
\hline$\tilde{h}=0, \quad v \neq 0$ & $\Psi_{l m}^{(4)}$ \\
\hline
\end{tabular}

\section{Acknowledgements}

This work was partially supported by the DGI under grant no. FIS2005-00752. A.E. acknowledges the financial support of the Spanish Ministry of Education through an FPU scholarship.

[1] Auberson G., Jain S.R., Khare A., A class of $N$-body problems with nearest- and next-to-nearest-neighbour interactions, J. Phys. A: Math. Gen., 2001, V.34, 695-724, cond-mat/0004012.

[2] Azuma H., Iso S., Explicit relation of the quantum Hall effect and the Calogero-Sutherland model, Phys. Lett. B, 1994, V.331, 107-113, hep-th/9312001.

[3] Baker T.H., Forrester P.J., The Calogero-Sutherland model and generalized classical polynomials, Comm. Math. Phys., 1997, V.188, 175-216, solv-int/9608004.

[4] Bogomolny E.B., Gerland U., Schmit C., Models of intermediate statistics, Phys. Rev. E, 1999, V.59, R1315R1318.

[5] Brink L., Turbiner A., Wyllard N., Hidden algebras of the (super) Calogero and Sutherland models, J. Math. Phys., 1998, V.39 1285-1315, hep-th/9705219.

[6] Calogero F., Solution of the one-dimensional $N$-body problem with quadratic and/or inversely quadratic pair potentials, J. Math. Phys., 1971, V.12, 419-436.

[7] Deguchi T., Ghosh P.K., Spin chains from super-models, J. Phys. Soc. Japan, 2001, V.70, 3225-3237, hep-th/0012058.

[8] Desrosiers P., Lapointe L., Mathieu P., Supersymmetric Calogero-Moser-Sutherland models and Jack superpolynomials, Nucl. Phys. B, 2001, V.606, 547-582, hep-th/0103178.

[9] D'Hoker E., Phong D.H., Calogero-Moser systems in SU(N) Seiberg-Witten theory, Nucl. Phys. B, 1998, V.513, 405-444, hep-th/9709053.

[10] Dunkl C.F., Differential-difference operators associated to reflection groups, Trans. Amer. Math. Soc., 1989, V.311, 167-183.

[11] Dunkl C.F., Orthogonal polynomials of types $A$ and $B$ and related Calogero models, Comm. Math. Phys., 1998, V.197, 451-487, q-alg/9710015.

[12] Enciso A., Finkel F., González-López A., Rodríguez M.A., Haldane-Shastry spin chains of $B C_{N}$ type, $N u c l$. Phys. B, 2005, V.707, 553-576, hep-th/0406054.

[13] Enciso A., Finkel F., González-López A., Rodríguez M.A., Solvable scalar and spin models with nearneighbors interactions. Phys. Lett. B, 2005, V.605, 214-222, hep-th/0407274.

[14] Enciso A., Finkel F., González-López A., Rodríguez M.A., Exchange operator formalism for $N$-body spin models with near-neighbors interactions, nlin.SI/0604073. 
[15] Ezung M., Gurappa N., Khare A., Panigrahi P.K., Quantum many-body systems with nearest and next-tonearest neighbor long-range interactions, Phys. Rev. B, 2005, V.71, 125121(8), cond-mat/0007005.

[16] Finkel F., Gómez-Ullate D., González-López A., Rodríguez M.A., Zhdanov R., $A_{N}$-type Dunkl operators and new spin Calogero-Sutherland models, Comm. Math. Phys., 2001, V.221, 477-497, hep-th/0102039.

[17] Finkel F., Gómez-Ullate D., González-López A., Rodríguez M.A., Zhdanov R., New spin Calogero-Sutherland models related to $B_{N}$-type Dunkl operators, Nucl. Phys. B, 2001, V.613, 472-496, hep-th/0103190.

[18] Gorsky A., Nekrasov N., Hamiltonian systems of Calogero type, and two dimensional Yang-Mills theory, Nucl. Phys. B, 1994, V.414, 213-238, hep-th/9304047.

[19] Haldane F.D.M., O(3) nonlinear $\sigma$ model and the topological distinction between integer- and half-integerspin antiferromagnets in two dimensions, Phys. Rev. Lett., 1988, V.60, 635-638.

[20] Heckman G.J., Dunkl operators, Astérisque, 1997, V.245, 223-246.

[21] Hitchin N., Stable bundles and integrable systems, Duke Math. J., 1987, V.54, 91-114.

[22] Jain S.R., Khare A., An exactly solvable many-body problem in one dimension and the short-range Dyson model, Phys. Lett. A, 1999, V.262, 35-39, cond-mat/9904121.

[23] Kasman, A., Bispectral KP solutions and linearization of Calogero-Moser particle systems, Comm. Math. Phys., 1995, V.172, 427-448, hep-th/9412124.

[24] Kirillov A.A., Jr., Lectures on affine Hecke algebras and Macdonald's conjectures, Bull. Amer. Math. Soc. (N.S.), 1997, V.34, 251-292, math.QA/9501219.

[25] Moser J., Three integrable Hamiltonian systems connected to isospectral deformations, Adv. Math., 1975, V.16, 197-220.

[26] Olshanetsky M.A., Perelomov A.M., Quantum integrable systems related to Lie algebras, Phys. Rep., 1983, V.94, 313-403.

[27] Perelomov A.M., Remark on the completeness of the coherent state system, Theor. Math. Phys., 1971, V.6, 213-224.

[28] Polychronakos A.P., Nonrelativistic bosonization and fractional statistics, Nucl. Phys. B, 1989, V.324, 597622 .

[29] Polychronakos A.P., Exchange operator formalism for integrable systems of particles, Phys. Rev. Lett., 1992, V.69, 703-706, hep-th/9202057.

[30] Polychronakos A.P., Lattice integrable systems of Haldane-Shastry type, Phys. Rev. Lett., 1993, V.70, 2329-2332, hep-th/9210109.

[31] Polychronakos A.P., Exact spectrum of an $\mathrm{SU}(N)$ spin chain with inverse-square exchange, Nucl. Phys. B, 1994, V.419, 553-566.

[32] Polychronakos A.P., Waves and solitons in the continuum limit of the Calogero-Sutherland model, 1995, Phys. Rev. Lett., 1995, V.74, 5153-5156, hep-th/9411054.

[33] Rühl W., Turbiner A.V., Exact solvability of the Calogero and Sutherland models, Mod. Phys. Lett. A, 1995, V.10, 2213-2221, hep-th/9506105.

[34] Shastry B.S., Exact solution of an $S=1 / 2$ Heisemberg antiferromagnetic chain with long-ranged interactions, Phys. Rev. Lett., 1988, V.60, 639-642.

[35] Shifman M.A., Turbiner A.V., Quantal problems with partial algebraization of the spectrum, Comm. Math. Phys., 1989, V.126, 347-365.

[36] Shukla P., Non-hermitian random matrices and the Calogero-Sutherland model, Phys. Rev. Lett., 2001, V.87, 194102(5).

[37] Sutherland B., Exact results for a quantum many-body problem in one dimension, I, II, Phys. Rev. A, 1971, V.4, 2019-2021, 1972, V.5, 1372-1376.

[38] Turbiner A.V., Quasi-exactly solvable problems and sl(2) algebra, Comm. Math. Phys., 1988, V.118, 467474.

[39] Turbiner A.V., Lie algebras and polynomials in one variable, J. Phys. A: Math. Gen., 1992, V.25, L1087L1093.

[40] Turbiner A.V., Lie algebras and linear operators with invariant subspaces, in Lie Algebras, Cohomologies and New Findings in Quantum Mechanics, Editors N. Kamran and P.J. Olver, Contemporary Mathematics, Vol. 160, Providence, AMS, 1994, 263-310, funct-an/9301001.

[41] Weyl H., The classical groups, Princeton, Princeton University Press, 1997.

[42] Yamamoto T., Multicomponent Calogero model of $B_{N}$-type confined in a harmonic potential, Phys. Lett. A, 1995, V.208, 293-302, cond-mat/9508012. 